\section{Habilidades cognitivas em indivíduos muito idosos: um estudo longitudinal}

\author{
Cognitive abilities in older seniors: \\ a longitudinal study
}

Irani I. de Lima Argimon 1 Lilian Milnitsky Stein 1

\title{
Introdução
}

1 Faculdade de Psicologia, Pontifícia Universidade Católica do Rio Grande do Sul, Porto Alegre, Brasil.

Correspondência I. I. L. Argimon Pós-graduação em Psicologia, Faculdade de Psicologia, Pontifícia Universidade Católica do Rio Grande do Sul. Av. Ipiranga 6681, prédio 11, 9o andar, Porto Alegre, RS 90619-900, Brasil. argimoni@pucrs.br

\begin{abstract}
Longitudinal studies of older seniors (over 80 years) are rare in the literature. This research was designed to study cognitive abilities in the oldest old at two different moments with a threeyear interval. The research was quantitative, with a longitudinal and prospective design. The sample consisted of 66 individuals from 80 to 95 years of age, randomly selected for phase $I$, with 46 of these individuals tested again three years later, in phase II. The instruments used were: Geriatric Depression Scale, Subjective Perception of Memory Problems, Mini-Mental State Examination, Digit Span, Buschke Test of Free and Cued Recall, and the Verbal Fluency TestAnimal Category. The results indicated that there was a small downward trend in the older seniors' performance at the end of the three-year period. More leisure activities and more years of formal schooling were good predictors of smaller decline in cognitive performance during the three-year period. Despite their age, older seniors in this study showed only a slight decline in their cognitive abilities, with little impact on their cognitive patterns.
\end{abstract}

Aged; Neuropsychology; Longevity; Cognition
Ao longo dos últimos anos, a população brasileira vem passando por um processo de transição demográfica, caracterizado pelo aumento da população idosa. O envelhecimento da população brasileira é um fenômeno crescente que necessita ser mais estudado, a fim de assegurar uma melhor qualidade de vida para essa população.

O processo de envelhecimento pode ser acompanhado pelo declínio das capacidades tanto físicas, como cognitivas dos idosos, de acordo com suas características de vida. Schaie 1, em um estudo longitudinal da população geral acima de sessenta anos, observou que nenhum dos participantes do estudo evidenciou um declínio generalizado em todas as habilidades cognitivas examinadas. Constatou-se que o declínio desencadeado pelo envelhecimento incidiu, especialmente, nas tarefas que exigiam rapidez, atenção, concentração e raciocínio indutivo.

Para Gorman \& Campbell 2, o déficit cognitivo em idosos consiste em lentidão leve, generalizada e perda de precisão, quando estes são comparados com pessoas mais jovens, e pode ser medido por testes objetivos que relacionem situações do cotidiano. Um nível educacional elevado mostrou-se, segundo esses autores, preventivo para o embotamento do estado mental durante o envelhecimento normal. 
As mudanças cognitivas precoces relacionadas ao início de demência e as mudanças do envelhecimento normais foram acompanhadas por Rubin et al. ${ }^{3}$. Os seus achados mostraram que pessoas idosas normais mantêm desempenho cognitivo estável quando medidos de forma longitudinal por avaliação clínica cuidadosa e testagem cognitiva repetida. Essa estabilidade tende a ser mantida, a menos que desenvolvam uma doença demencial, quando, então, um declínio agudo do desempenho é observado.

A avaliação das características de idosos que ficam no limite entre envelhecimento normal e patológico foi realizada por Ritchie et al. 4 . Esses autores examinaram, por testagem computadorizada, a função cognitiva em idosos saudáveis, identificando entre eles um subgrupo com déficit cognitivo subclínico. Também foram realizados exames neurológicos padronizados e os genótipos de apolipoproteína $\mathrm{E}$ (Apo E). Na avaliação transversal para algumas habilidades cognitivas, tais como linguagem e memória secundária, não foram detectadas diferenças relacionadas à idade dos participantes, enquanto outras apresentaram deterioração na análise transversal e na longitudinal. Um nível inicial de QI mais alto mostrou efeito protetor de demência para pessoas acima de 75 anos. Pessoas com mais anos de escolaridade apresentaram estabilidade temporal na linguagem e tarefas de memória secundária, mas mostraram prejuízo nas tarefas viso-espaciais, semelhante ao detectado naquelas de baixa escolaridade. Cinco padrões de déficit subclínico foram isolados, e dois grupos com perfis clínicos diferentes (dos quais apenas um manifestou o alelo Apo E4) mostraram risco aumentado para desenvolver demência. Uma alta prevalência de depressão também foi encontrada. O envelhecimento não afetou toda a cognição uniformemente e a alta escolarização inicial parece ter adiado o declínio para certas tarefas cognitivas. Padrões distintos de modificações cognitivas foram observados na demência inicial, nas mudanças benignas e nas relacionadas à doença depressiva.

\section{Objetivo}

Estudos longitudinais com idosos acima de oitenta anos são escassos na literatura, o que é justificado, principalmente, pela perda amostral característica dessa faixa etária. Tendo em vista essa limitação da literatura, o presente es- tudo pretendeu contribuir com uma análise longitudinal de idosos em idade bastante avançada. A pesquisa foi delineada com o objetivo de analisar as modificações no perfil de algumas habilidades cognitivas em indivíduos muito idosos em um período de três anos. As habilidades cognitivas enfocadas foram: percepção subjetiva da memória, fluência verbal, memória e atenção. Além disso, foi investigado se escolaridade, idade e lazer contribuíam para explicar diferenças nos escores observados no intervalo de três anos.

\section{Método}

Considerando-se os objetivos propostos, o enfoque metodológico utilizado foi de uma pesquisa do tipo quantitativo, com delineamento longitudinal e prospectivo, ou seja, os idosos foram examinados em dois momentos, com um intervalo de três anos, em janeiro de 1998 (etapa I) e reexaminados em 2001 (etapa II), utilizando-se os mesmos instrumentos.

\section{Amostra}

Para a etapa I do estudo, escolheu-se, aleatoriamente, uma amostra de $35,0 \%$ (sorteio de 77 indivíduos de uma população de 219) dos idosos com mais de oitenta anos residentes no $\mathrm{Mu}$ nicípio de Veranópolis, na serra gaúcha (conforme registro no Instituto Brasileiro de Geografia e Estatística 5). A grande maioria da população era de trabalhadores rurais descendente de italianos.

Fizeram parte da população-alvo somente os idosos que residiam no município em janeiro de 1998 e tinham oitenta anos completos ou mais naquela data, sendo incluídos somente casos com idade documentada. Na amostra de 77 idosos, quatro recusaram-se a participar do estudo. Outros quatro idosos apresentaram critérios do Diagnostic and Statistical Manual of Mental Disorders - Fourth Edition (DSM-IV) 6 para demência e dois apresentaram critérios para delirium. Um indivíduo apresentou intensa ansiedade durante a entrevista de coleta de dados, e não pôde ser estudado. A amostra, na etapa I, ficou, então, composta por 66 idosos. A proporção amostral de $36,0 \%$ idosos do sexo masculino (24 homens) e de $64,0 \%$ idosos do sexo feminino (42 mulheres) não diferiu da proporção na população total de pessoas aci ma de oitenta anos de Veranópolis. O tempo mé- 
dio de escolaridade era de 2,6 anos de estudo; $38,0 \%$ dos idosos residiam na área rural do município e 62,0\% residiam na área urbana.

No intervalo de três anos, dos 66 idosos da etapa I, 17 foram a óbito, dois se negaram a participar do seguimento da pesquisa e um estava viajando, ficando, portanto, a amostra final da etapa II composta por 46 idosos residentes na comunidade de Veranópolis, sendo 35 $(76,1 \%)$ do sexo feminino e $11(23,9 \%)$ do sexo masculino. A idade média dos idosos na segunda etapa foi de 87 anos ( $D P=2,92$ ). A idade dos idosos do sexo feminino variou de 84 a 95 anos, com uma idade média de 87 anos ( $D P=2,77$ ), enquanto a idade dos idosos do sexo masculino variou de 83 a 94 anos, com uma idade média de 87 anos (DP $=3,52$ ).

\section{Instrumentos}

Com base na literatura, foi organizado um conjunto de instrumentos validados para nossa população e que tem sido referência em estudos atuais 7 . Em um segundo momento, essa seleção de instrumentos foi submetida ao julgamento de três juízes, especialistas em psicodiagnóstico, para que pudessem opinar sobre qual seria a melhor seqüência de aplicação dos instrumentos, respeitando as características de cada um.

Os instrumentos foram utilizados na seguinte ordem: Questionário de Dados Sócio-Demográficos; Percepção Subjetiva de Queixas da Memória 8; Span de Números 9; Mini-Exame do Estado Mental (MEEM) 10; Teste de Buschke Lembranças Livres e com Pistas 11; Fluência VerbalCategoria Animal 12; Escala de Depressão Geriátrica 13 . Os instrumentos foram respondidos sem cansaço e com facilidade de entendimento das instruções em um período que variou de uma hora e dez minutos à uma hora e meia.

\section{Procedimentos da coleta de dados}

A coleta de dados foi realizada por uma psicóloga em uma sessão individual. Os idosos que residiam na central urbana do município foram testados em espaço cedido pela Prefeitura de Veranópolis. Já para aqueles que moravam na zona rural, a sessão de coleta de dados ocorreu em suas próprias residências.

$\mathrm{Na}$ entrevista individual, o participante era inicialmente informado dos objetivos da pes quisa. Para facilitar possíveis contatos posteriores, foram solicitadas informações sobre endereço e telefone, que constaram em uma ficha de dados sócio-demográficos. Os aspectos éticos foram devidamente considerados e condi- cionados aos requisitos contidos nas Diretrizes e Normas Regulamentadoras de Pesquisa Envolvendo Seres Humanos, do Conselho Nacional de Saúde, sendo o estudo submetido à apreciação e aprovado pelo Comitê de Ética da Pontifícia Universidade Católica do Rio Grande do Sul (PUCRS).

A ordem de aplicação dos instrumentos obedeceu ao critério de primeiro utilizar os instrumentos que mediam a atenção, em seguida os de memória, resolução de problemas, vocabulário e escala de depressão (conforme orientação dos juízes consultados).

\section{Procedimentos estatísticos}

para a análise dos dados

As análises estatísticas foram realizadas por meio do programa SPSS para Windows, versão 11.5. Estatísticas descritivas foram inicialmente utilizadas para a elaboração de um perfil sócio-demográfico da amostra. Foram feitos vários testes paramétricos, sendo considerados significativos quando o valor de p era $\bullet 0,05$, como segue: para a comparação do desempenho dos idosos nos dois momentos de coleta de dados (etapa I e etapa II), com um intervalo de três anos, utilizou-se o t Student - para amostras pareadas. Já o Coeficiente de Correlação de Spearman foi empregado para avaliar o grau de associação entre a variável Percepção Subjetiva de Queixas de Memória e os resultados objetivos no teste de memória Lembranças Livres $e$ com Pistas, bem como entre o número de opções de lazer que os idosos desenvolviam e seu desempenho nos testes de habilidades.

Foram também realizadas análises de variância (ANOVA para medidas repetidas) a fim de investigar o efeito do número de anos de escolaridade no desempenho cognitivo dos idosos, comparando as etapas I e II.

Tendo por base as análises realizadas, partiu-se para uma pesquisa exploratória, segundo procedimentos sugeridos por Altman 14, com o objetivo de investigar se escolaridade, lazer e depressão poderiam explicar a variação no desempenho cognitivo dos idosos no intervalo de três anos. Para isso, geraram-se modelos preditivos do desempenho cognitivo dos idosos para as variáveis Delta (que indicam a variação da diferença entre os escores apresentados na etapa I e na etapa II). Para essa análise, foram utilizados os escores acima de zero na etapa II. Para testagem desses modelos, foi utilizada a Análise Multivariada de Regressão Linear, utilizando o método Enter. 


\section{Resultados}

Pelo caráter longitudinal do presente estudo, os resultados serão apresentados tomando por base somente aqueles idosos que compunham ambas as etapas, sendo apresentados em duas partes: (1) caracterização sócio-demográfica da amostra da etapa II e (2) análise comparativa das habilidades cognitivas dos idosos, em um intervalo de três anos.

\section{Características sócio-demográficas}

Na etapa II do estudo, entre os idosos examinados, houve alguns participantes que, por problemas clínicos, tais como visão, audição entre outros, não conseguiram completar todos os instrumentos propostos. Portanto, responderam ao MEEM 38 participantes; ao Lembranças Livres e com Pistas, 35; ao Percepção Subjetiva de Queixas de Memória, 44; ao Span de Números, 41; ao Fluência Verbal, 44. Dois idosos encontravam-se acamados, com muita dificuldade de visão e audição, por isso suas contribuições ficaram limitadas aos dados sócio-demográficos.

Em relação ao estado civil, $31(67,4 \%)$ dos idosos eram viúvos; 10 (21,7\%) eram casados, 3 $(6,5 \%)$, solteiros; 1 (2,2\%), separado; 1 não respondeu. A distribuição dos anos de escolaridade foi a seguinte: $27(58,7 \%)$ idosos tinham até três anos de escolaridade e 19 (41,3\%) tinham de quatro a nove anos de escolaridade. A média de anos de escolaridade foi de 2,54 (DP = 2,61 ), caracterizando uma escolarização básica incompleta. Quanto à capacidade de leitura, 32 $(69,6 \%)$ dos idosos sabiam ler e 31 deles $(67,4 \%)$ sabiam escrever. Dos idosos pesquisados, $62,0 \%$ residiam na zona urbana.

Quanto ao fator familiar relacionado a que pessoas moravam com o idoso, $26(56,5 \%)$ deles residiam com filhos, 7 (15,2\%) com o cônjuge, 7
$(15,2 \%)$ viviam com outros parentes (netos, sobrinhos, irmãos) e 6 (13,0\%) moravam sozinhos.

Quanto ao hábito de fumar, foi observado que $40(87,0 \%)$ dos idosos nunca fumaram, 4 $(8,7 \%)$ pararam de fumar havia mais de cinco anos e $2(4,3 \%)$ ainda fumavam. Em relação à bebida alcoólica, o vinho foi eleito pelos participantes como sendo a bebida de sua preferência e $25(54,3 \%)$ tomavam em média um cálice de vinho nas refeições (almoço e jantar).

Quando solicitados a avaliar a saúde, 24 $(52,2 \%)$ idosos responderam "regular", 14 (30,4\%) responderam "ruim" e $8(17,4 \%)$ avaliaram-na como "boa".

Quanto ao tipo de atividades de preferência dos sujeitos em seus momentos de lazer, a maior preferência recaía nas atividades religiosas, seguidas por assistir a programas de televisão e ouvir rádio. Atividades físicas relacionadas com caminhadas, cuidar de hortas, pescaria, jogos de bocha e sinuca também tiveram a opção de mais de 50,0\% dos idosos entrevistados.

\section{Habilidades cognitivas}

A Tabela 1 apresenta os resultados das comparações do desempenho dos idosos nos testes de habilidades cognitivas, relacionadas com a linguagem, memória e atenção, num intervalo de três anos.

Em uma avaliação cognitiva dos idosos pelo MEEM (Tabela 1), observou-se que houve uma pequena tendência de decréscimo no desempenho cognitivo geral em um período de três anos (ainda que somente marginalmente significativa).

Com relação à suspeita de demência, examinando-se os escores apresentados no MEEM e utilizando-se o ponto de corte empregado em outras pesquisas brasileiras 15,16 para diferenciar idosos saudáveis daqueles com sinais de demência inicial de 13, no caso de idosos sem

Desempenho dos idosos nas avaliações das habilidades cognitivas nas duas etapas.

\begin{tabular}{lrrrrrr}
\hline Instrumentos & \multicolumn{2}{c}{ Etapa I } & \multicolumn{2}{c}{ Etapa II } & \multirow{2}{*}{$\mathbf{t}$} & P \\
& M & DP & M & DP & \\
& & & & & \\
\hline Mini-Exame Estado Mental & 21,08 & 4,40 & 19,66 & 5,24 & 1,947 & 0,059 \\
Lembranças Livres e com Pistas & 92,20 & 8,52 & 81,86 & 24,94 & 2,972 & 0,005 \\
Percepção Subjetiva Queixas de Memória & 25,70 & 3,84 & 27,82 & 5,23 & $-2,342$ & 0,024 \\
Span de Números & 6,29 & 2,04 & 4,61 & 2,31 & 5,476 & 0,001 \\
Fluência Verbal & 10,80 & 3,89 & 9,98 & 3,76 & 1,429 & 0,160 \\
\hline
\end{tabular}


escolaridade, e 18, no caso de idosos com até sete anos de escolaridade, observou-se que nenhum dos pesquisados obteve um escore abaixo de 13 na etapa I. Após o intervalo de três anos, constatou-se que apenas três idosos $(6,5 \%)$ tiveram um decréscimo em seu desempenho no MEEM, situando-se, então, abaixo do ponto de corte 13. Ainda que os parâmetros estabelecidos pelos pontos de corte aqui utilizados devam ser vistos com cautela, uma vez que outros patamares já foram sugeridos por outras pesquisas com população brasileira 17, os nossos achados com indivíduos muito idosos parecem sugerir que o percentual de participantes com provável demência é menor do que os encontrados em estudos epidemiológicos $(12,0 \%$ a $39,0 \%) 18,19$.

Com relação à memória, na comparação da pontuação apresentada em Lembranças Livres e com Pistas no teste de Buschke nas duas etapas (conforme dados da segunda linha da Tabela 1), observou-se que houve uma diminuição significativa nos escores médios dos idosos no período de três anos. Mesmo havendo um declínio na habilidade da memória, ele não é suficiente para caracterizar uma demência que, segundo Petersen et al. 20 seria identificada por um escore menor de 69. Na amostra estudada, somente $13,0 \%$ dos idosos apresentaram escores inferiores a 69 na etapa II.

A percepção dos idosos sobre problemas com sua memória (Tabela 1) tendeu a aumentar com o passar do tempo. Conforme o esperado, eles percebiam ter mais problemas de memória do que há três anos. Uma questão a ser investigada era se essa percepção subjetiva de dificuldades mnemônicas correspondia ao desempenho desses idosos no teste de memória. Correlacionando o resultado obtido na Percepção Subjetiva de Queixas de Memória e os resultados objetivos no teste de memória Lembranças Livres e com Pistas, constatou-se que um número mais elevado de queixas de problemas de memória não necessariamente correspondia a mais problemas de memória, na medida em que não houve uma correlação significativa entre esses resultados, tanto na etapa $\mathrm{I}(\mathrm{r}=-0,27, \mathrm{p}>0,05)$, quanto na etapa II $(\mathrm{r}=-0,26$, $\mathrm{p}>0,05)$.

No que tange a habilidades atencionais (Tabela 1, quarta linha), observou-se que, no intervalo de três anos, os idosos apresentaram um decréscimo significativo no seu desempenho. Os dados sugerem um prejuízo nas habilidades cognitivas de atenção e memória de trabalho, que, segundo Groth-Marnat 21, Lesak 22, Wechsler 23, já seria indicativo na etapa II de um sinal de demência.
Conforme Tabela 1, na habilidade relacionada à fluência verbal, pode-se observar que não houve diferença estatisticamente significativa dos escores no intervalo de três anos. Usando o ponto de corte 9 no teste de fluência verbal para diferenciar indivíduos saudáveis de indivíduos com declínio cognitivo 24 , o teste exato de Fisher indicou uma associação ( $\mathrm{p}<$ 0,05 ) entre os escores em fluência verbal das etapas I e II. Utilizando-se os resíduos ajustados padronizados, verificou-se que os idosos que possuíam um escore inferior ao ponto de corte 9 na etapa I mantiveram, em geral, esse valor três anos após, o mesmo ocorrendo com os idosos que tiveram um escore igual ou maior que 9 , os quais igualmente se mantiveram no mesmo nível de desempenho. Isso indica que, de fato, não parece ter ocorrido um declínio dos escores em fluência verbal nos idosos avaliados.

Para investigar se havia alguma relação entre o número de opções de lazer que os idosos desenvolviam e seu desempenho nas habilidades cognitivas avaliadas (somente para a etapa II), foi calculado o coeficiente de correlação de Spearman para verificar o grau de associação entre o número de atividades de lazer e os escores nos testes. Os resultados indicaram que parece haver uma correlação positiva entre o número de atividades de lazer e o desempenho cognitivo dos indivíduos, especificamente: MEEM $(\mathrm{r}=0,48, \mathrm{p}<0,01)$, Lembranças Livres e com Pistas $(\mathrm{r}=0,37, \mathrm{p}<0,05)$ e Fluência Verbal $(\mathrm{r}=$ $0,55, \mathrm{p}<0,01$ ). Os resultados sugerem que um melhor desempenho nesses instrumentos de avaliação cognitiva está associado a um número mais elevado de atividades de lazer. Ainda com relação ao lazer, avaliou-se a associação entre sintomas de depressão e número de atividades de lazer. Conforme o esperado, esta relação é significativa e inversa $(r=-0,42, p<0,01)$.

Para testar a hipótese de que mais anos de escolaridade serviriam de fator protetor no declínio das habilidades cognitivas, os idosos foram divididos em dois grupos: um com até três anos de escolaridade e outro com quatro ou mais anos de escolaridade. Os efeitos principais das análises de variância independentes da escolaridade, relativos à comparação do desempenho dos idosos nas duas etapas do estudo, não serão aqui apresentados, haja vista que já foram analisados anteriormente (Tabela 1). Os outros resultados obtidos nas ANOVAs foram os seguintes:

No desempenho no MEEM, evidenciou-se um efeito principal para escolaridade $\left(\mathrm{F}_{(1,36)}=\right.$ $7,213, \mathrm{p}<0,05)$. Os idosos com menos anos de escolaridade tiveram um resultado significati- 
vamente inferior no $\mathrm{MEEM}(\mathrm{M}=18,81$; $\mathrm{DP}=$ $0,85)$, quando comparados com idosos com mais anos de escolaridade $(\mathrm{M}=22,34$; $\mathrm{DP}=$ $0,99)$, independentemente da etapa de testagem $\left(\mathrm{F}_{(1,36)}<0,001, \mathrm{p}>0,05\right)$.

Quanto à habilidade da atenção, avaliada por meio do teste Span de Números, evidenciouse um efeito principal para escolaridade $\left(\mathrm{F}_{(1,39)}\right.$ $=17,244, \mathrm{p}<0,001)$. Os idosos com até três anos de escolaridade obtiveram um resultado significativamente inferior $(\mathrm{M}=4,60$; $\mathrm{DP}=0,32)$, quando comparados com idosos de quatro ou mais anos de escolaridade $(\mathrm{M}=6,78$; $\mathrm{DP}=0,41)$.

No que diz respeito a lembranças livres e com pistas, quando se comparou o desempenho dos idosos com até três anos de escolaridade ao dos idosos com quatro anos ou mais de escolaridade, a ANOVA não evidenciou diferença significativa $\left(\mathrm{F}_{(1,33)}=1,521, \mathrm{p}>0,05\right)$. $\mathrm{O}$ mesmo ocorreu na comparação da percepção subjetiva de problemas de memória $\left(\mathrm{F}_{(1,42)}=\right.$ $2,233, p>0,05)$ e na de habilidade de linguagem através do teste de fluência verbal $\left(\mathrm{F}_{(1,42)}=\right.$ $0,480, \mathrm{p}>0,05)$.

A testagem de possíveis modelos preditivos para a variação do desempenho cognitivo dos idosos, comparando-se as etapas I e II, é apresentada na Tabela 2.

Os modelos de regressão apontam uma contribuição independente de sintomas depressi- vos na predição da diferença apresentada entre as duas etapas no desempenho no MEEM. Os dados sugerem que o incremento de sintomas depressivos nos idosos estava associado a um pior desempenho no MEEM.

A adequabilidade do modelo de análise de regressão para avaliar a variação do desempenho do MEEM é bastante boa, já que o coeficiente de determinação global obtido (R2) indica que $31,0 \%$ da variância do MEEM conseguem ser explicados pelas variáveis depressão, lazer e escolaridade. Já os outros modelos propostos não apontam para contribuições preditivas relativas à variação do desempenho nos testes de Buschke, Span de Números e Fluência Verbal.

\section{Discussão}

A especificidade do presente estudo está relacionada ao desenvolvimento cognitivo em idosos com mais de oitenta anos. Apesar de terem sido detectadas algumas perdas no desempenho de algumas habilidades cognitivas estudadas nesse intervalo de três anos, elas não foram suficientes para caracterizar um declínio cognitivo que pudesse ser considerado compatível com uma demência.

Todavia, a considerável perda amostral na segunda etapa do estudo, ainda que esperada

Tabela 2

Modelos de regressão linear para o desempenho cognitivo dos idosos, utilizando a diferença dos escores da etapa I e da etapa II.

\begin{tabular}{|c|c|c|c|c|c|c|}
\hline \multirow[t]{2}{*}{ Testes cognitivos } & \multirow[t]{2}{*}{$\mathbf{R}^{2}$} & \multirow[t]{2}{*}{ Preditores } & \multirow[t]{2}{*}{$\mathbf{P}$} & \multicolumn{2}{|c|}{$\begin{array}{l}\text { Coeficiente não } \\
\text { padronizado }\end{array}$} & \multirow{2}{*}{$\begin{array}{c}\text { Coeficiente } \\
\text { padronizado } \\
\text { Beta }\end{array}$} \\
\hline & & & & Beta & Erro padrão & \\
\hline Delta & $0,310^{\star}$ & Escolaridade & 0,054 & 0,409 & 0,204 & 0,312 \\
\hline Mini-Exame do & & Lazer & 0,078 & $-0,122$ & 0,438 & $-0,050$ \\
\hline Estado Mental & & Depressão & 0,007 & 0,465 & 0,159 & 0,520 \\
\hline \multirow[t]{4}{*}{ Delta Buschke } & 0,127 & Escolaridade & 0,538 & $-0,578$ & 0,928 & $-0,112$ \\
\hline & & Lazer & 0,487 & $-1,316$ & 1,870 & $-0,133$ \\
\hline & & Perc. Subjet. Memória & 0,189 & 0,858 & 0,638 & 0,252 \\
\hline & & Depressão & 0,554 & 0,521 & 0,871 & 0,113 \\
\hline \multirow[t]{3}{*}{ Delta Span de Números } & 0,067 & Escolaridade & 0,190 & $-0,172$ & 0,129 & $-0,234$ \\
\hline & & Lazer & 0,353 & 0,228 & 0,242 & 0,181 \\
\hline & & Depressão & 0,649 & 4,8340 & 0,105 & 0,087 \\
\hline \multirow[t]{3}{*}{ Delta Fluência Verbal } & 0,104 & Escolaridade & 0,388 & $-0,217$ & 0,249 & $-0,146$ \\
\hline & & Lazer & 0,358 & $-0,464$ & 0,499 & $-0,172$ \\
\hline & & Depressão & 0,557 & 0,118 & 0,199 & 0,109 \\
\hline
\end{tabular}

${ }^{*} p<0,01$. 
em virtude da idade muito avançada dos participantes, bem como as características singulares da população estudada (zona serrana gaúcha de cultura italiana e agrícola) podem vir a limitar as considerações mais conclusivas que se possa fazer. Apesar dessas limitações inerentes ao estudo, as avaliações realizadas com os idosos estudados indicaram que eles ainda conservavam muito de suas condições cognitivas depois de três anos, embora tivessem oitenta anos ou mais. Sendo assim, os resultados obtidos com o MEEM, não obstante esse teste ser uma forma de "rastreio" amplo das condições cognitivas, foram corroborados no presente estudo pelas avaliações de habilidades cognitivas feitas por intermédio dos outros instrumentos. É possível, então, levantar a hipótese de que algumas características sócio-demográficas da população estudada, por exemplo: o envolvimento com a comunidade, diferentes atividades de lazer, convívio com familiares e atividades físicas, podem atuar como fatores de proteção ao declínio cognitivo. Outro aspecto que também parece contribuir como mais um fator de proteção do declínio cognitivo é a escolaridade. Os idosos que tinham mais anos de escolaridade conservaram um melhor resultado no período de três anos em muitas das funções cognitivas examinadas.

A depressão é um problema de saúde freqüente entre as pessoas de muita idade, embora a identificação seja muitas vezes difícil na prática clínica. É possível supor que indivíduos com mais de oitenta anos tenham uma saúde física mais debilitada que os indivíduos com menos idade. Pode-se inferir que exista um maior risco de apresentarem sintomas depressivos, não em decorrência da idade mais avançada, mas por causa do estado de saúde mais precário.

No que tange ao efeito de outras variáveis, além do tempo de três anos, quais sejam escolaridade, número de atividades de lazer e depressão, os resultados parecem indicar que outros fatores não examinados também têm uma contribuição importante. Ao mesmo tempo, a partir da aceitação do princípio de que a cultura e a evolução cultural podem afetar o desenvolvimento cognitivo, é possível levar em conta a plasticidade de que os idosos mostram ser capazes, tais como adaptação a novas situações que a vida lhes oferece e a possibilidade de pensar em estratégias que sirvam de fatores protetores em seu desenvolvimento cognitivo.

Sabe-se que o esquecimento é um fator comum nas pessoas de diferentes faixas etárias; o que não é comum é que as pessoas não aproveitem pistas para recordar-se de eventos pas- sados. Estudos como de Haninnen et al. 25 e Petersen et al. 20 mostram que o não-aproveitamento de pistas seria compatível com uma demência inicial. No que diz respeito à habilidade de memória para figuras através de lembranças livres e com pistas, decorrido o período de três anos, o desempenho médio aferido denotou uma queda, ainda que esta não tenha sido suficientemente acentuada para caracterizar uma demência inicial. Assim sendo, levando-se em conta a idade avançada do grupo de participantes estudados, parece haver uma boa preservação da sua capacidade de lembranças e aproveitamento de pistas. Contudo, esses idosos subestimaram suas próprias habilidades mnemônicas quando fizeram uma avaliação subjetiva de suas capacidades. Clinicamente, esses resultados sugerem que mais queixas de problemas de memória não são, necessariamente, um retrato fidedigno das habilidades mnemônicas dos idosos. Assim, uma apreciação subjetiva da memória deve ser considerada com cautela pelo clínico e corroborado por outros indicadores mais objetivos.

No que diz respeito à influência da escolaridade sobre o desempenho da atenção, avaliado através do teste de Span de Números, o trabalho de Osterweil et al. 26 não mostrou um efeito da escolaridade no desempenho de idosos de diferentes faixas etárias. Não alinhado com esses resultados, o presente estudo observou diferenças significativas no desempenho dos idosos dependendo de seu nível de escolaridade formal, indicando que idosos com mais anos de escolaridade formal mostraram um melhor resultado no teste de atenção do que os idosos com menos escolaridade. Achados semelhantes a estes também foram encontrados por Argimon 27, em um estudo transversal com idosos. O que pode explicar essas discrepâncias de resultados é que a população estudada por este e por outros trabalhos difere em, pelo menos, vinte anos de idade a mais do que aquela investigada por Osterweil et al. 26. Uma hipótese a ser testada por investigações futuras é se o possível efeito protetor da escolaridade contra um declínio cognitivo é mais evidente quanto mais avançada a idade dos indivíduos.

$\mathrm{Na}$ habilidade da linguagem, o resultado apresentado não foi diferente do encontrado na literatura 28,29 , ou seja, esta foi uma das habilidades que se manteve preservada ao longo dos três anos do estudo e independente da escolaridade dos idosos. Esses resultados vêm reforçar uma prática diagnóstica usual 22,30 para avaliação cognitiva na terceira idade, haja vista que, no desenvolvimento das habilidades cognitivas dessa faixa etária, aquela ligada à lin- 
guagem é mais resistente à degeneração pela idade, sendo por isso muito utilizada como indicador pré-mórbido na avaliação de condições cognitivas dos idosos. Por conseguinte, um desempenho prejudicado na área de lingua gem tem sido utilizado como um marcador de uma demência em andamento.

Os idosos do presente estudo, pertencentes a uma comunidade específica, ainda conservavam determinada autonomia, sem muitas mudanças nas atividades do dia-a-dia, e participavam de uma rede familiar e social sólida. Um número maior de atividades realizadas por esses indivíduos na vida diária, tais como envolvimento com amigos, com a igreja e a cultura existente no local, bem como atividades físicas, por exemplo caminhadas, seja como exercício, seja para deslocamento habitual, parece ter servido como um fator protetor de suas condições cognitivas. Dados epidemiológicos 31 referem, como uma dimensão imprescindível para uma boa qualidade de vida, a auto-suficiência no cuidado consigo mesmo, destacando a importância da manutenção de diferentes opções de

\section{Resumo}

Estudos longitudinais com idosos com mais de oitenta anos são escassos na literatura. A pesquisa foi delineada com o objetivo de analisar as modificações no perfil de algumas habilidades cognitivas em indivíduos muito idosos, em dois momentos, com um intervalo de três anos. Pesquisa do tipo quantitativo, com delineamento longitudinal e prospectivo. A amostra, randômica, foi constituída por 66 indivíduos de 80 a 95 anos de idade na etapa I e, três anos após, na etapa II, por 46 idosos. Os instrumentos usados foram: Escala de Depressão Geriátrica, Questionário de Percepção Subjetiva de Queixas de Memória, Mini-Exame do Estado Mental, Span de Números, Teste de Lembranças Livres e com Pistas de Buschke, Teste de Fluência Verbal - Categoria Animal. Houve uma pequena tendência de decréscimo no desempenho cognitivo em um período de três anos. Maior número de atividades de lazer e mais anos de escolaridade foram fatores preditivos de menor variação no desempenho cognitivo. Apesar da idade avançada, os idosos apresentaram um desempenho de habilidades cognitivas cujo declínio foi de intensidade leve, não sendo suficiente para acarretar mudanças significativas no seu padrão cognitivo.

Idosos; Neuropsicologia; Longevidade; Cognição atividades de lazer como um fator de maior autonomia e bem-estar emocional.

Apesar da idade avançada, esses idosos apresentaram um desempenho de habilidades cognitivas cujo declínio é de intensidade leve, não sendo suficiente para acarretar mudanças significativas no seu padrão cognitivo.

Por isso, pode-se dizer que o envelhecimento é um processo em que, para cada pessoa, as mudanças físicas, comportamentais e sociais desenvolvem-se em ritmos diferentes, sendo a idade cronológica apenas um dos aspectos, entre outros, que podem ou não afetar o bem-estar do idoso. Muitos fatores psicossociais que contribuem para um envelhecimento saudável incluem família, educação, cuidados com a própria saúde, além de motivação e iniciativa da própria pessoa muito idosa. Acredita-se que, tendo-se mais subsídios sobre o processo do envelhecimento saudável, mais se poderá trabalhar para a prevenção de uma demência, como também para identificar precocemente sinais iniciais desta.

\section{Colaboradores}

I. I. L. Argimon contribuiu na aplicação dos instrumentos, organização do banco de dados, e L. M. Stein contribuiu no delineamento e acompanhamento do estudo. A elaboração do artigo ficou a cargo de ambas as autoras, na mesma proporção de trabalho e responsabilidades.

\section{Agradecimentos}

Agradecemos ao Instituto de Geriatria e Gerontologia da Pontifícia Universidade Católica do Rio Grande do Sul e à Prefeitura e Comunidade de Veranópolis. 


\section{Referências}

1. Schaie KW, Willis SL. Psychometric intelligence and aging. In: Blanchard-Fieds E, Hess TM, editors. Perspectives on cognitive change in adulthood and aging. New York: McGraw Hill; 1996. p. 293-322.

2. Gorman WF, Campbell CD. Mental acuity of the normal elderly. J Okla State Med Assoc 1995; 88:119-23.

3. Rubin EH, Storandt M, Miller JP, Kinscherf DA, Grant EA, Morris JC, et al. A prospective study of cognitive function and onset of dementia in cognitively healthy elders. Arch Neurology 1998; 55:359-401.

4. Ritchie K, Touchon J, Ledesert B, Leibovici D, Gorce AM. Establishing the limits and characteristics of normal age-related cognitive decline. Rev Epidemiol Sante Publique 1997; 45:373-81.

5. Instituto Brasileiro de Geografia e Estatística. Dados preliminares da população no Brasil. Rio de Janeiro: Instituto Brasileiro de Geografia e Estatística; 2002.

6. American Psychiatric Association. Diagnostic and statistical manual of mental disorders - fourth edition (DSM-IV). Washington DC: American Psychiatric Association; 1994.

7. Xavier FMF. Prevalência de declínio cognitivo associado ao envelhecimento em uma população de idosos com mais de 80 anos, residentes na comunidade [Dissertação de Mestrado]. São Paulo: Universidade Federal de São Paulo; 1999.

8. Crook TH, Feher EP, Larrabee GJ. Assessment of memory complaints. In: Age-associated memory impairment: the MAC-Q. Intern Psychogeriatry 1992; 4:165-76.

9. Wechsler D. WAIS-R - Wechsler adult intelligence scale-revised. Cleveland: Psychological Corporation; 1981.

10. Folstein MF, Folstein SE, McHugh PR. "Mini-Mental State": a practical method for grading the cognitive state of patients for the clinician. J Psych Res 1975; 12:189-98.

11. Buschke H, Fuld PA. Evolution of storage, retention and retrieving in disordered memory and learning. Neurology 1974; 11:1019-25.

12. Spreen O, Benton AL. Neurosensory Center Comprehensive Examination for Aphasia (NCCEA). Victoria: University of Victoria; 1977.

13. Yesavage JA, Brink TL, Rose TL, Lum O, Huang V, Adey M, et al. Development and validation of a geriatric depression screening scale: a preliminary report. J Psychiatr Res 1983; 49:37-49.

14. Altman DG. Pratical statistics for medical reseach. London: Chapman \& Hall/CRC Press; 1991.

15. Bertolucci PHF, Brucki SMD, Campacci S, Juliano Y. Mini-exame do estado mental e escolaridade. Arq Neuropsiquiatr 1994; 52:1-7.

16. Laks J, Batista EMR, Guilherme ERL, Contino ALB, Faria MEV, Figueira I, et al. O mini-exame do estado mental de uma comunidade: dados parciais de Santo Antônio de Pádua, Rio de Janeiro. Arq Neuropsiquiatr 2003; 61:782-5.

17. Almeida OP. Mini-exame do estado mental e o diagnóstico de demência no Brasil. Arq Neuropsiquiatr 1998; 56:605-12.
18. Henderson S. Epidemiologia dos transtornos psiquiátricos. In: Kaplan HI, Sadock BJ, organizadores. Tratado de psiquiatria. 6a Ed. Porto Alegre: Artmed; 1999. p. 2721-7.

19. Howieson DB, Dame A, Camicioli R, Sexton G, Payami H, Kaye A. Marcadores cognitivos que precedem a demência de Alzheimer em pacientes muito idosos sadios. Demência - Programa de Educação Continuada 1998; 1:1-58.

20. Petersen RC, Smith G, Kokmen E, Ivnik RJ, Tangalos EG. Memory function in normal aging. Neurology 1992; 42:396-401.

21. Groth-Marnat G. Wechsler adult intelligence scaleIII supplement. In: Groth-Marnat G, editor. Handbook of psychological assessment. $3^{\text {rd }}$ Ed. New York: Wiley \& Sons; 1999. p. 132-204.

22. Lesak MD. Neuropsychological assessment. New York: Oxford University Press; 1995.

23. Wechsler D. WMS-R. Wechsler memory scale-revised manual. San Antonio: Psychological Corporation; 1987.

24. Brucki SM, Malheiros SMF, Okamoto IV, Bertolucci PHF. Dados normativos para o uso do teste Fluência Verbal (categoria animal), em nosso meio Arq Neuropsiquiatr 1997; 55:56-61.

25. Hanninen T, Koivisto K, Reinikainen KJ, Helkala EL, Soininen H, Mykkanen L, et al. Prevalence of ageing-associated cognitive decline in elderly population. Age Ageing 1996; 25:201-5.

26. Osterweil D, Mulford P, Syndulko K, Martin M. Cognitive function in old and very old residents of a residential facility: relationship to age, education and dementia. J Am Geritr Soc 1994; 42: 766-73.

27. Argimon IL. Desenvolvimento cognitivo na terceira idade [Tese de Doutorado]. Porto Alegre: Faculdade de Psicologia, Pontifícia Universidade Católica do Rio Grande do Sul; 2002.

28. Welsh KA, Butters N, Mohs RC, Beekly D, Edland $\mathrm{S}$, Fillenbaum G, et al. The consortium to establish a registry for Alzheimer's disease (CERAD). Neurology 1994; 44:609-14.

29. Wiederholt WC, Cahn D, Butters NM, Salmon DP, Silverstein DK, Connor EB. Effects of age, gender and education on selected neuropsychological tests in an eldery community cohort. J Am Geriatr Soc 1993; 41:639-47.

30. Cunha JA. Inventário de depressão de Beck: catálogo de técnicas úteis. In: Cunha JA, organizador. Psicodiagnóstico. 5a Ed. Porto Alegre: Artmed; 2000. p. 239-42.

31. Forlenza OV, Caramelli P. Neuropsiquiatria geriátrica. São Paulo: Atheneu; 2001.

Recebido em 02/Fev/2004

Versão final reapresentada em 23/Jun/2004 Aprovado em 17/Ago/2004 